\title{
Analysis and Statistics of the Spectroscopic Sample of Byurakan-IRAS Galaxies
}

\author{
Gohar S. Harutyunyan and Areg M. Mickaelian \\ Byurakan Astrophysical Observatory (BAO), Armenia \\ Email: goharutyunyan@gmail.com, aregmick@yahoo.com
}

\begin{abstract}
A summary and general analysis of optical spectroscopic data for 255 Byurakan-IRAS Galaxies (BIG) obtained with BAO 2.6m, SAO 6m, OHP 1.93m telescopes, as well as SDSS DR7, DR8, and DR9 is given. The BIG sample is the result of optical identifications of IRAS PSC sources at high-galactic latitudes using the First Byurakan Survey (FBS) low-dispersion spectra. Among the 1178 objects most are spiral galaxies and there is a number of ULIRGs. All but one have emission lines; we have discovered 68 AGN and composite spectrum objects among them and the others are mostly Starburst Galaxies (SB). All possible physical characteristics have been measured and/or calculated, including physical sizes and optical and IR/FIR luminosities. The masses have been estimated based on mass-luminosity relations for spiral galaxies. As it appears, most of these objects are giant massive galaxies. Various multiwavelength (MW) data have been retrieved from recent catalogues from X-ray to radio and MW SEDs have been built, which have been matched to their optical classifications. Luminosity evolution of these objects has been studies.
\end{abstract}

Keywords. IR galaxies, spectroscopy, AGN, Starburst galaxies

Since 1995 the project of optical identifications of IRAS point sources using the First Byurakan Survey (FBS, Markarian et al. 1989) low-dispersion spectra is being carried out (Mickaelian 1995). The purpose is to obtain a homogeneous sample of optical counterparts for IR sources, as well as reveal new galaxies with active nuclei (AGN), starburst galaxies (SB), interacting pairs and galaxies with high IR luminosity (Ultra-Luminous InfraRed Galaxies, ULIRG). Identified 1178 galaxies are called Byurakan-IRAS Galaxies (BIG). A spectroscopic survey for BIG objects was carried out with three telescopes: $2.6 \mathrm{~m}$ of the Byurakan Astrophysical Observatory (BAO, Armenia), 6m of the Special Astrophysical Observatory (SAO, Russia) and 1.93m of Observatoire de Haut-Provence (OHP, France) (Mickaelian \& Sargsyan 2010). Most of these objects are spiral galaxies and there is a number of ULIRGs among them. Objects having spectroscopic observations with the mentioned telescopes, as well as those having SDSS spectra (DR7, DR8, and DR9, Ahn et al. 2013) make up the sample of 255 spectroscopically observed BIG objects.

Spectroscopic studies of BIG object have several objectives:

- spectroscopic follow-up for all brighter $\left(<18^{m}\right)$ objects of the sample,

- discovery and study of new AGN and SB,

- discovery and study of new ULIRGs,

- deep imagery of selected objects and the empty fields to reveal new ULIRG/HLIRG,

- 2D spectroscopy of interacting/merging systems,

- search for obscured IRAS galaxies (using SST and other deeper data),

- study of starburst/AGN/interaction phenomena and their interrelationship. 

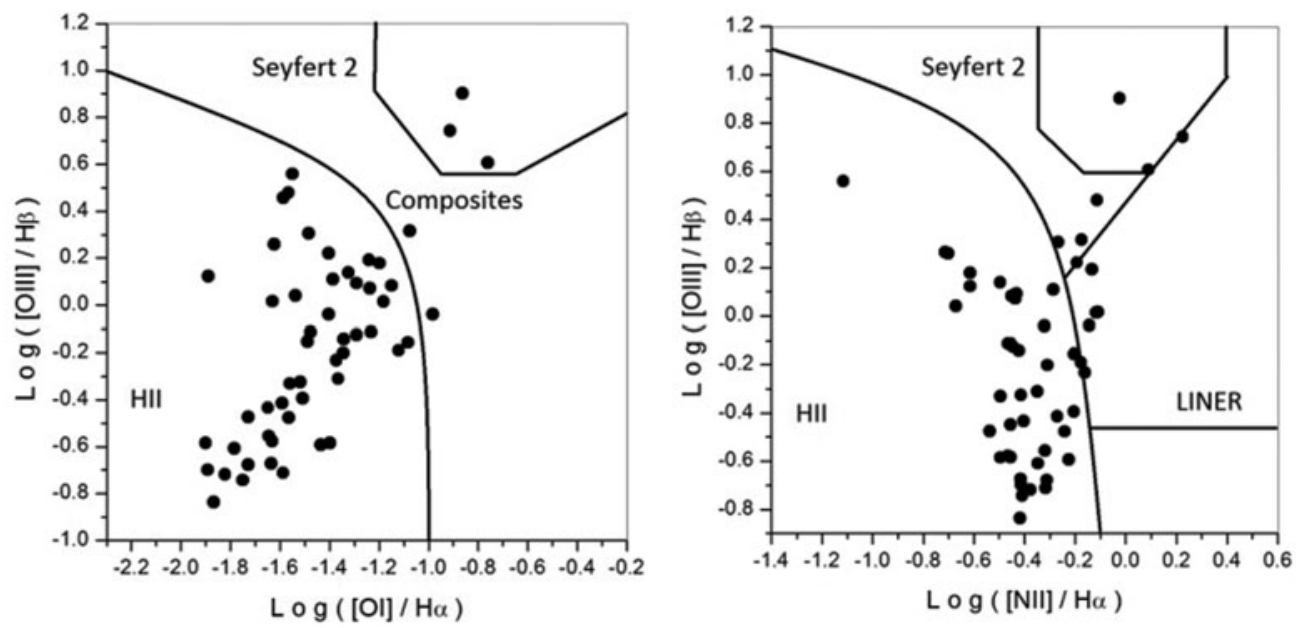

Figure 1. Diagnostic diagrams for BIG objects based on SDSS spectral classification.

Fig. 1 gives diagnostic diagrams (Veilleux \& Osterbrock 1987) for the BIG sample that have been used for narrow emission-line galaxies for classification by activity types. Classification by eye has been carried out to compare with the one by diagnostic diagrams as well, as not all objects appear on them due to weakness of some emission lines. All spectral lines have been taken into account.

In our spectroscopic sample of 255 BIG objects, we have $43(17 \%)$ AGN, $25(10 \%)$ composite spectrum objects, 157 (62\%) SB, 29 (11\%) emission-line galaxies without a definite type, and 1 absorption-line galaxy. All possible physical characteristics have been measured and/or calculated, including radial velocities and distances, angular and physical sizes, absolute magnitudes and luminosities (both optical and IR). IR luminosities and star formation rates have been calculated from the IR fluxes (Duc et al. 1997). Additional available date were taken from Catalogue of QSOs and Active Galaxies (Veron-Cetty \& Veron 2010).

There are 3 ULIRGs in our sample. The average redshift is $\mathrm{z}=0.06743$ and the average distance, $269 \mathrm{Mpc}$. The masses of BIG objects have been estimated based on mass-luminosity relations for spiral galaxies. As it appears, most of these objects are giant massive galaxies. To make a complete study of these galaxies possible, we have retrieved various multiwavelength (MW) data for the full sample of 1178 objects from recent catalogues from X-ray to radio. MW SEDs have been built, which have been matched to their optical classifications. Star-formation rates have been calculated to compare to their other physical characteristics, such as morphology, activity types, UV, optical, IR and radio luminosities, etc.

\section{References}

Duc, P. A., Mirabel, I. F., \& Maza, J. 1997, A\&্AS, 124, 533

Markarian, B. E., Lipovetski, V. A., Stepanian, J. A., Erastova, L. K., \& Shapovalova, A. I. 1989, Com. SAO 62, 5

Mickaelian, A. M. 1995, Ap 38, 625

Mickaelian, A. M. \& Sargsyan, L. A. 2010, Ap 53, 483

Veilleux, S. \& Osterbrock, D. E. 1987, ApJS 63, 295

Veron-Cetty M. P. \& Veron P. 2010, A\&A 518, A10 